\title{
Exact calculations of the paranematic interaction energy for colloidal dispersions in the isotropic phase of a nematogenic material
}

\author{
J.-B. Fournier风 \\ Laboratoire de Physico-Chimie Théorique, E. S. P. C.I., \\ 10 rue Vauquelin, F-75231 Paris cedex 05, France \\ P. Galatolai \\ LBHP, Université Paris 7-Denis Diderot, Case 7056, \\ 2 place Jussieu, F-75251 Paris cedex 05, France
}

(Dated: November 13, 2018)

\begin{abstract}
In a recent paper [Phys. Rev. E 61, 2831 (2000)], Borštnik, Stark and Žumer have studied the stability of a colloidal dispersion of micron-sized spherical particles in the isotropic phase of a nematogenic material. Close to the nematic transition, the attraction due to a surface-induced paranematic order can yield flocculation. Their calculation of the nematic-mediated interaction was based on an ansatz for the order-parameter profile. We compare it with an exact numerical calculation, showing that their results are qualitatively correct. Besides, we point out that in the considered regime, the exact interaction is extremely well approximated by a simple analytical formula which is asymptotically exact.
\end{abstract}

PACS numbers: 82.70.Dd, $61.30 . \mathrm{Cz}$

In recent years, a large interest has been devoted to understanding the interactions and phase behavior of colloidal particles dispersed in a nematic phase [1, 2, 3, 4, 5] or in the isotropic phase of a nematogenic compound 6 , 7, 8, 9. In the nematic phase, colloids experience a specific elastic interaction because they induce competing distortions of the nematic director field. New physics arises due to the long-range character of this interaction and the induction of topological defects [1].

In the isotropic phase, the surface of colloidal particles can induce a local paranematic order 10, 11], giving rise to a short-range elastic interaction [6, 7]. Two effects compete: an attraction due to the favorable overlapping of the paranematic halos and a repulsion due to the distortion of the director field. For small particles, of size comparable to the nematic-isotropic coherence length $\xi$, it has been predicted that repulsion may dominate and stabilize the colloidal dispersion [6, 9]. (Note that latex particles as small as $50 \mathrm{~nm}$ have been successfully dispersed in lyotropic nematics 12].) On the other hand, Borštnik, Stark and Žumer have predicted that for micron-sized particles attraction dominates [7], which allows to trigger flocculation close to the nematic transition 8 .

The results of Borštnik, Stark and Žumer [8] are based on a composite ansatz for the nematic director field $\mathbf{n}$ and for the scalar order-parameter $Q$, within an uniaxial hypothesis. It turns out that our exact calculations [9, based on a multipolar expansion for the full tensorial order-parameter $Q_{i j}$, rest on the same theoretical model, and can be performed also for micron-sized particles.

\footnotetext{
*Electronic address: ibf@turner.pct.espci.fr
}

${ }^{\dagger}$ Electronic address: galatola@ccr.jussieu.fr

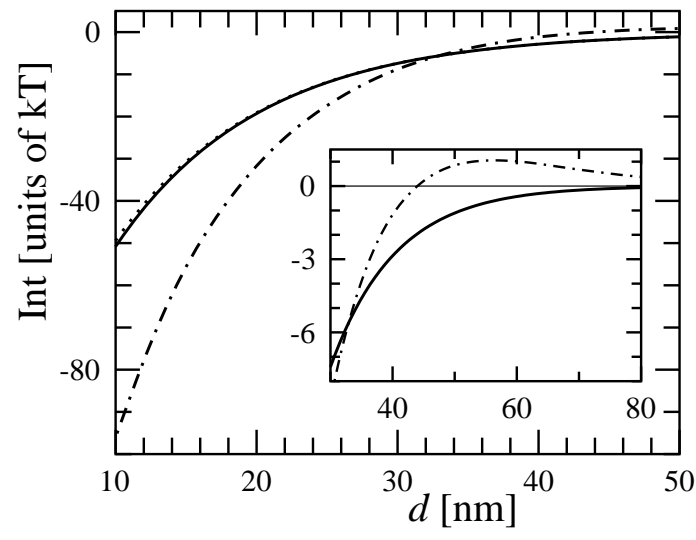

FIG. 1: Paranematic interaction energy between two spherical particles of radius $R=0.25 \mu \mathrm{m}$ as a function of their distance to contact $d$. The parameters are $a=1.8 \times$ $10^{5} \mathrm{~J} \mathrm{~m}^{-3} \mathrm{~K}^{-1}, T^{*}=313.5 \mathrm{~K}, \Delta T=1.3 \mathrm{~K}, L_{1}=9 \times$ $10^{-12} \mathrm{~J} \mathrm{~m}^{-1}, Q_{s}=0.3, G_{Q}=G_{n}=10^{-3} \mathrm{~J} \mathrm{~m}^{-2}$. The corresponding nematic coherence length is $\xi=10.7 \mathrm{~nm}$. The dashed-dotted line is extracted from Fig. 7 of Ref. [7]. The full-line is the exact result, numerically calculated according to Ref. 91. The dotted-line, practically coincident with the full-line, corresponds to the asymptotic formula (8).

In this Brief Report, we check the correctness of the paranematic interaction energy used in Ref. [8], by comparing it with the exact one, numerically calculated according to the method of Ref. [9]. For the typical values considered in Ref. [8], we find that the exact interaction is attractive instead of repulsive at distances $\gtrsim 5 \xi$, and that it is about two times weaker at distances of the order of $\xi$ (Fig. 1). However, the order of magnitude and the sign of the interaction is correct in the regime where the paranematic attraction competes with the electrostatic repulsion. In particular, at particles' separations of 
the order of $\xi$, the paranematic attraction remains much larger than the van der Waals attraction: this implies that the conclusions of Ref. [8] regarding critical flocculation phenomena remain qualitatively correct. Finally, we show that in the whole regime of interest, the exact interaction calculated numerically is extremely well approximated by a simple asymptotic formula [see Eq. (8)].

The Landau-de Gennes 13 paranematic free-energy density considered in Refs. [7, 8] is

$$
f=\frac{1}{2} a \Delta T Q^{2}+\frac{3}{2} L_{1}(\nabla Q)^{2}+\frac{9}{2} L_{1} Q^{2}|\nabla \mathbf{n}|^{2}
$$

for the bulk, and

$$
f_{s}=G_{Q}\left(Q-Q_{s}\right)^{2}+3 G_{n} Q Q_{s} \sin ^{2} \theta
$$

for the surface. Here $Q$ is the scalar order-parameter and $\mathbf{n}$ the nematic director. The parameter $Q_{s}$ is the orderparameter favored by the particles' surface, and $\theta$ is the angle between the direction of $\mathbf{n}$ at the surface and the normal to the surface $\boldsymbol{\nu}$, which is assumed to correspond to the easy-axis. The coefficients $a$ and $L_{1}$ are material parameters, $\Delta T=T-T^{\star}$ is the difference between the actual temperature $T$ and the limit of stability $T^{\star}$ of the isotropic phase, $G_{Q}$ and $G_{n}$ are introduced to describe the strength of the surface anchoring.

Incidentally, we note that the surface free-energy density (2) is inconsistent within a Landau-de Gennes framework, unless $G_{Q}=G_{n}$. Indeed, at quadratic order, the most general expansion in terms of the tensorial order parameter $Q_{i j}$ and of the normal $\boldsymbol{\nu}$ to the surface can be written as

$$
\begin{aligned}
f_{s}= & g_{1} Q_{i j} \nu_{i} \nu_{j}+g_{21} Q_{i j} Q_{i j}+g_{22} Q_{i j} Q_{j k} \nu_{i} \nu_{k} \\
& +g_{23} Q_{i j} Q_{k l} \nu_{i} \nu_{j} \nu_{k} \nu_{l}
\end{aligned}
$$

where summation over repeated indices is implied. For a uniaxial tensorial order-parameter

$$
Q_{i j}=\frac{3}{2} Q\left(n_{i} n_{j}-\frac{1}{3} \delta_{i j}\right)
$$

this yields

$$
\begin{aligned}
f_{s}= & g_{1} Q+\frac{1}{2}\left[3 g_{21}+2\left(g_{22}+g_{23}\right)\right] Q^{2} \\
& -\frac{3}{4}\left[2 g_{1} Q+\left(g_{22}+4 g_{23}\right) Q^{2}\right] \sin ^{2} \theta \\
& +\frac{9}{4} g_{23} Q^{2} \sin ^{4} \theta .
\end{aligned}
$$

Matching Eqs. (5) and (2) requires setting $g_{23}=0$ and consequently $g_{22}=0$. Equation (5) then becomes

$$
f_{s}=-\frac{g_{1}^{2}}{6 g_{21}}+\frac{3}{2} g_{21}\left[\left(Q-Q_{s}\right)^{2}+3 Q Q_{s} \sin ^{2} \theta\right],
$$

with $Q_{s}=-g_{1} / 3 g_{21}$. Therefore, Eq. (2) is compatible with this expression only if $G_{Q}=G_{n}$.

With the required condition $G_{Q}=G_{n} \equiv G$, the freeenergy considered in Ref. [8] is identical to the one we used in Ref. [9], with the correspondence [14]:

$$
\begin{aligned}
L^{\dagger} & =2 L_{1}, \quad S^{\dagger}=\frac{3}{2} Q, \quad S_{0}^{\dagger}=\frac{3}{2} Q_{s}, \\
a^{\dagger} & =\frac{2}{3} a \Delta T, \quad W^{\dagger}=\frac{4}{3} G,
\end{aligned}
$$

where we have dagged the quantities appearing in Ref. [9]. With the above relationships, we have numerically recalculated the exact interaction energy between two spherical particles of radius $R$ as a function of their distance to contact $d$-using the same parameters as in Ref. [7]. The comparison with the results of the ansatz of Borštnik, Stark and Žumer [7] is shown in Fig. 1]. We find a qualitative agreement, as previously discussed. Note that the exact nematic director profile displays a Saturn-ring defect [9], which is absent in the ansatz of Ref. [7]. For the case of micron-sized particles considered in Refs. [7] 8], this defect lies however in a region where the paranematic is almost completely melted.

Finally, we have compared our numerical calculation with the asymptotic formula obtained by us in Ref. [9]. With the correspondence (7), the latter reads

$$
F=-48 \pi L_{1} \xi\left(\frac{Q_{s}}{A}\right)^{2} \frac{e^{-\bar{d}}}{2 \bar{R}+\bar{d}}
$$

where $\bar{d}=d / \xi, \bar{R}=R / \xi$, and $A$ is a constant given by

$$
A=\frac{27 \bar{\ell}}{\bar{R}^{4}}+\frac{6+27 \bar{\ell}}{\bar{R}^{3}}+\frac{6+12 \bar{\ell}}{\bar{R}^{2}}+\frac{2+3 \bar{\ell}}{\bar{R}}
$$

where $\bar{\ell}=L_{1} / G \xi$ is the reduced extrapolation length of the anchoring. The nematic coherence length is $\xi=$ $\sqrt{3 L_{1} / a \Delta T}$. As shown in Fig. 1, the agreement between the numerical and the analytical calculations is excellent in the range of separations relevant to the colloidal flocculation discussed in Ref. [8]. Owing to its simplicity and validity, formula (8) thus offers a straightforward means to systematically investigate the stability of such paranematic-wetted colloids. 
[1] P. Poulin, H. Stark, T. C. Lubensky, and D. A. Weitz, Science 275, 1770 (1997).

[2] P. Poulin, V. Cabuil, and D. A. Weitz, Phys. Rev. Lett. 79, 4862 (1997).

[3] H. Stark, J. Stelzer, and R. Bernhard, Eur. Phys. J. B 10, 515 (1999).

[4] R. Yamamoto, Phys. Rev. Lett. 87, 075502 (2001).

[5] D. Andrienko, G. Germano, and M. P. Allen, Phys. Rev. E 63, 041701 (2001).

[6] P. Galatola and J.-B. Fournier, Mol. Crys. Liq. Cryst. 330, 535 (1999).

[7] A. Borštnik, H. Stark, and S. Žumer, Phys. Rev. E 60, 4210 (1999).
[8] A. Borštnik, H. Stark, and S. Žumer, Phys. Rev. E 61, 2831 (2000).

[9] P. Galatola and J.-B. Fournier, Phys. Rev. Lett. 86, 3915 (2001).

[10] P. Sheng, Phys. Rev. Lett. 37, 1059 (1976).

[11] K. Miyano, Phys. Rev. Lett. 43, 51 (1979).

[12] O. Mondain-Monval, J. C. Dedieu, T. Gulik-Krzywicki, and P. Poulin, Eur. Phys. J. B 12, 167 (1999).

[13] P.-G. de Gennes and J. Prost, The Physics of Liquid Crystals (Clarendon, Oxford, 1993).

[14] Actually, in our free-energy we keep the full biaxial orderparameter $Q_{i j}$ instead of assuming the uniaxial order (4). 